\title{
The Method for Fault Diagnosis of Electric Actuator for a Type of Self-propelled Rocket Launcher Based on the Fuzzy Clustering
}

\author{
Xu Bing, Wang Hong-li, Wang Han- Bing, Liu Fu-Li \\ Dept. of 5, Wuhan Mechanical Technology, Wuhan 430075, China \\ email:1712178221@qq.com.cn
}

Keywords: rocket launcher; fuzzy clustering; faulty diagnosis; fuzzy relation

\begin{abstract}
Fuzzy theory is a forceful analytic tool to the faulty diagnosis of complicated system. A fuzzy clustering method is introduced on the basis of original fault diagnosis. The initial sort is acquired by fuzzy equivalent matrix and F-statistic, and the initial iterative matrix for the fuzzy c-means is also acquired. So it gets the most optimize class matrix and the fuzzy clustering center. The practical example shows the result calculated by fuzzy clustering is good agreement with the actual inspections, and the method can be applied to the faulty diagnosis of electric operation system of self-propelled rocket launcher. It can distinguish complicated faults more efficiently and accurately, and it has a good foreground.
\end{abstract}

\section{Foreword}

Electric actuator applied to rocket launcher is an extremely complicated system, to the extent that battle effectiveness of rocket launcher will be impaired directly in case of failure with electric operation system, which further leads to degraded technical supporting capacity of the army. In practice, a faulty rocket is usually disused by storing in depot, this not only prevents the army from routine drilling, but also substantially weakens its battle effectiveness.

In this article, the theory of fuzzy clustering analysis is applied in a comprehensive manner, standard fault samples taken and data collected from practical operation for testing are used as classified samples, fault diagnosis of electric actuator for rocket launcher is conducted with transitive closure method based on fuzzy equivalent matrices and fuzzy C-means-based classification, which is proved to bring out crucial significance and remarkable military benefit in improving battle performance as well as technical condition and level of rocket launcher.

\section{Fundamental \& Methodology for Fault Diagnosis with Fuzzy Clustering}

Basic Thinking.The method applied herein is that, by assuming normal operation of tested system, a set of data can be tested at first. Depending upon system functions, internal correlation exists among these sampling points, thus the method of fuzzy clustering may be applied in analyzing sampling points to derive standard functional model for normal system. Then, fuzzy clustering of tested values is conducted during actual operation of the system; in case of system fault, clustering center surely offsets standard model under normal condition, and the fault with those system functions as corresponding membership can be diagnosed on the basis of analysis and calculation of fuzzy distance. Obviously, this is a method of model identification in comparison with standard model.

Clustering Method.

\section{1) Data Standardization}

Assume domain of discourse $U=\left\{x_{1}, x_{2}, \cdots, x_{n}\right\}$ as the objects to be classified, where $x_{1}=a_{1}, x_{2}=a_{2}, \cdots, x_{n 1}=a_{n 1}, x_{n 1+1}=b_{1}, x_{n 1+2}=b_{2}, \cdots, x_{n}=b_{n 2}, a_{1}, a_{2}, \cdots, a_{n 1}=$ standard fault sample, $b_{1}, b_{2}, \cdots, b_{n 2}=$ fault sample to be tested; each object consists of $m$ indexes of characteristic for expression of its properties, $x_{i}=\left(x_{i 1}, x_{i 2}, \cdots, x_{i m}\right)(i=1,2, \cdots, n)$; then, original data matrix is derived; 
and then, standardization processing is performed with original data matrix derived. Usually, standard data is compressed to interval[0,1], with range transformation:

\section{2) Rating (Construct Fuzzy Similar Matrix)}

$$
x_{i k}^{\prime}=\frac{x_{i k}-\min _{1 \leq i \leq n}\left\{x_{i k}\right\}}{\max _{1 \leq i \leq n}\left\{x_{i k}\right\}-\min _{1 \leq i \leq n}\left\{x_{i k}\right\}}
$$

Construct fuzzy similar matrix $R=\left(\gamma_{i j}\right)$, where $\gamma_{i j}=$ similar coefficient, to describe similarity between samples $i$ and $j$. Angle cosine is applied:

\section{3) Clustering}

$$
\gamma_{i j}=\left(\sum_{k=1}^{m} x_{i k} \cdot x_{j k}\right) /\left(\left(\sum_{k=1}^{m} x_{i k}^{2}\right) \cdot\left(\sum_{k=1}^{m} x_{j k}^{2}\right)\right)
$$

Clustering requires fuzzy relation $R$ to be fuzzy equivalent relation, fuzzy equivalent relation matrix $R$ can be derived from square root calculation,i.e. Derivation in sequence $R^{2}, R^{4}, \cdots, R^{2^{k}}$, until $R^{2^{k}}=R^{2^{k-1}}$, then $R=R^{2^{k}}$.

Determination of Best Threshold $\lambda$. Best value $\lambda$ is determined by $F$ - statistics; assume $c$ as number of classification corresponding to value $\lambda$, number of samples class $j$ is $n j$, and samples class $j$ are expressed as $x_{1}^{(j)}, x_{2}^{(j)}, \cdots, x_{n j}^{(j)}$, clustering center of samples class $j$ is vector $x^{(j)}=\left(\bar{x}_{1}^{(j)}, \bar{x}_{2}^{(j)}, \cdots, \bar{x}_{m}^{(j)}\right)$, where $\bar{x}_{k}^{(j)}=$ mean value of number $k$ characteristics, namely, $\bar{x}_{k}^{(j)}=\frac{1}{n j} \sum_{i=1}^{n j} x_{i k}^{(j)}(k=1,2, \cdots, m)$.

$$
F \text {-statistics: } \quad F=\frac{\sum_{j=1}^{n j} n j\left\|\bar{x}^{(j)}-\bar{x}\right\|^{2} /(r-c)}{\sum_{j=1}^{r} \sum_{j=1}^{n j} n j\left\|x_{i}^{(j)}-\bar{x}_{i}^{(j)}\right\|^{2} /(n-r)}
$$

Where $\left\|\bar{x}^{(j)}-\bar{x}\right\|=\sqrt{\sum_{k=1}^{m}\left(\bar{x}_{k}^{(j)}-\bar{x}^{(j)}\right)^{2}}=$ distance between $\bar{x}^{(j)}$ and $\bar{x},\left\|x_{i}^{(j)}-\bar{x}_{i}^{(j)}\right\|=$ distance between $x_{i}^{(j)}$ of samples class $j$ and $\operatorname{center} \bar{x}^{(j)}$.

$F$-Statistics is subjected to F-distribution with DOF of $c-1, n-$; numerator means distance between classes, and denominator means distance of samples in the same class; therefore, bigger $F$ value suggests bigger distance and bigger difference between classes for better classification.

\section{Fuzzy C-mean Clustering Algorithm.}

(1) Class $\mathrm{C}$ of samples can be derived from value $\lambda$ corresponding to $\mathrm{F}$ maximum, then classification is transformed to corresponding initial membership matrix $A_{c}^{(0)}$ before iterative algorithm.

(2) Calculate clustering center $V_{i}^{(q)}$ from $V_{i}=\sum_{k=1}^{n}\left(r_{i k}\right)^{\alpha} x_{k} / \sum_{i=1}^{m}\left(r_{i k}\right)^{\alpha} V_{i}^{(q)} \quad$ ( $q$ =iterative times).

(3) Calculate $r_{i j}^{*}$ based on $V_{i}^{(q)} \quad r_{i j}^{*}=\left[\sum_{k=1}^{m}\left\|x_{j}-V_{i}\right\| /\left\|x_{j}-V_{k}\right\|^{2 /(\alpha-1)}\right]^{-1}$ to derive new classification matrix $A_{c}^{*(q+1)}$, where $\alpha$ is taken to $1<\alpha<+\infty$.

(4) Randomly given a small positive number $\varepsilon$, and assume $\left\|A^{(q+1)}-A^{q}\right\|<\varepsilon$, iteration closes, otherwise, assume $q=q+1$ and go back to (2) for further iteration, and finally best classification matrix $A$ and clustering center $V$ are derived.

Analysis on the Conclusion of Fault Diagnosis.One best classification is derived after fuzzy clustering analysis. If a given sample to be tested belongs to the same class as standard sample of a fault, indicating occurrence of such fault; however, when samples to be tested belong to the same 
class as several classes of standard fault samples, indicating similarity of samples to be tested to several classes of standard fault samples, namely, possibility of concurrence of these faults. If samples to be tested do not belong to the same class as any class of standard fault, it indicates a new class of fault. By case analysis and diagnosis, standard fault samples can be improved further to increase confidence of fault diagnosis.

\section{Fault Diagnosis of Electric Actuator for Self-propelled Rocket Launcher}

For various systems of rocket launcher, and in occurrence of one phenomenon, it's likely to be a fault by many causes; likewise, multiple fault causes are likely to result in as much fault phenomena; for example, poor contact of moving contact for manual resistance controller is one cause of the fault that could lead to a number of faults with rocket, including unstable operation, abrupt shutdown, enlargement of motor output voltage surge, and etc; therefore, relation between fault and phenomenon is intricate. To determine main cause of certain phenomenon, the extent of relation between the cause of fault and phenomenon of fault should have to be identified at first, i.e. a weighing coefficient to be assessed based upon empirical and statistic results; working process of whole fuzzy diagnosis is shown in the chart below:

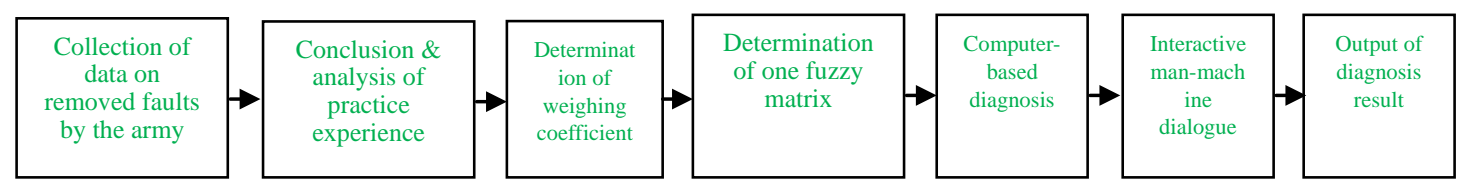

Chart 1 Fuzzy Diagnosis Process

Table 1 contains original data used for fault diagnosis based on fuzzy clustering.

Table 1. Original Data Standard fault sample (SFS)

\begin{tabular}{|c|c|c|c|c|c|c|c|}
\hline Sample & Characte & eristic & $\mathrm{C} \mathrm{Di}$ & strib & ution & & Type of Fault \\
\hline $\begin{array}{l}\text { Standard fault } \\
\text { sample } 1\end{array}$ & $\begin{array}{lll}4 & 2 & 2 \\
1 & 1 & 0\end{array}$ & $\begin{array}{ll}2 & 0 \\
0 & \end{array}$ & 0 & 3 & 3 & & $\begin{array}{l}\text { Controller fault } \\
\text { Control panel fault }\end{array}$ \\
\hline $\begin{array}{l}\text { Standard fault } \\
\text { sample } 2\end{array}$ & $\begin{array}{lll}0 & 2 & 4 \\
0 & & 1\end{array}$ & $\begin{array}{ll}4 & 0 \\
1 & \end{array}$ & 0 & 2 & 2 & 0 & Polarized relay failure \\
\hline $\begin{array}{l}\text { Standard fault } \\
\text { sample } 3\end{array}$ & $\begin{array}{lll}3 & 0 & 4 \\
0 & 0 & 2\end{array}$ & $\begin{array}{ll}4 & 3 \\
2 & \end{array}$ & 3 & 1 & 1 & & $\begin{array}{l}\text { Intermediary relay } \\
\text { failure }\end{array}$ \\
\hline $\begin{array}{l}\text { Standard fault } \\
\text { sample } 4\end{array}$ & $\begin{array}{lll}0 & 0 & 0 \\
0 & & 0\end{array}$ & $\begin{array}{ll}0 & 0 \\
0 & \end{array}$ & 0 & 2 & & 2 & \begin{tabular}{|ll} 
Variable & resistor \\
short-circuit &
\end{tabular} \\
\hline $\begin{array}{l}\text { Standard fault } \\
\text { sample } 5\end{array}$ & $\begin{array}{lll}0 & 0 & 0 \\
4 & 0 & 3\end{array}$ & $\begin{array}{ll}0 & 4 \\
3 & \end{array}$ & 0 & 0 & 0 & 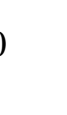 & $\begin{array}{ll}\text { Angle } & \text { retainer } \\
\text { malfunction } & \end{array}$ \\
\hline $\begin{array}{l}\text { Standard fault } \\
\text { sample } 6\end{array}$ & $\begin{array}{lll}1 & 0 & 0 \\
3 & 1 & 0\end{array}$ & $\begin{array}{ll}0 & 3 \\
0 & \end{array}$ & 2 & 1 & 1 & L & $\begin{array}{l}\text { Enlarged motor fault } \\
\text { Type of fault TBD }\end{array}$ \\
\hline $\begin{array}{ll}\text { Standard } & \text { fault } \\
\text { sample } 7 & \end{array}$ & $\begin{array}{lll}0 & 2 & 1 \\
0 & 2 & 0\end{array}$ & $\begin{array}{ll}1 & 1 \\
0 & \end{array}$ & 1 & 2 & 0 & & $\begin{array}{l}\text { Type of fault TBD } \\
\text { Type of fault TBD }\end{array}$ \\
\hline $\begin{array}{l}\text { Sample a to be } \\
\text { tested }\end{array}$ & $\begin{array}{lll}2 & 0 & 0 \\
3 & 0 & 0\end{array}$ & $\begin{array}{ll}0 & 3 \\
0 & \end{array}$ & 3 & 1 & 1 & & \\
\hline $\begin{array}{l}\text { Sample b to be } \\
\text { tested }\end{array}$ & $\begin{array}{lll}0 & 2 & 4 \\
0 & 0 & 1\end{array}$ & $\begin{array}{ll}4 & 0 \\
1 & \end{array}$ & 0 & 3 & 3 & & \\
\hline $\begin{array}{l}\text { Sample c to be } \\
\text { tested }\end{array}$ & $\begin{array}{lll}0 & 3 & 6 \\
0 & 0 & 2\end{array}$ & $\begin{array}{ll}6 & 0 \\
2 & \end{array}$ & 0 & 3 & 3 & & \\
\hline
\end{tabular}




$V=\left(\begin{array}{lllllll}0.0001 & 0.0000 & 0.9993 & 0.0342 & 0.0000 & 0.0000 & 0.1283 \\ 0.0000 & 0.0000 & 0.0001 & 0.0096 & 0.0001 & 1.0000 & 0.0300 \\ 0.0001 & 0.0000 & 0.0002 & 0.0233 & 0.9998 & 0.0000 & 0.0780 \\ 0.0001 & 0.0000 & 0.0002 & 0.8928 & 0.0000 & 0.0000 & 0.6539 \\ 0.9996 & 0.0000 & 0.0002 & 0.0371 & 0.0001 & 0.0000 & 0.1028 \\ 0.0000 & 1.0000 & 0.0000 & 0.0030 & 0.0000 & 0.0000 & 0.0070 \\ 0.0001 & 0.0000 & 0.0000 & 0.0000 & 0.0000 & 0.0000 & 0.0000\end{array}\right)$

Diagnosis outcome based on above analysis is that, data a to be tested indicates angle retainer malfunction, while data b and c to be tested indicate control panel fault, base on which engineering technician can work out method and steps for removal of such faults, so as to eliminate any faults discovered in a timely manner. It's obvious that this fuzzy clustering is easy for realization of real-time and intelligent fault diagnosis. Case examination suggests consistent theoretical calculation and on-site inspection. This is the evidence that fuzzy clustering is potential for practical application by providing a desirable approach in fault diagnosis of complex system.

\section{Conclusion}

In this discourse, a new method for fault diagnosis of electric actuator for self-propelled rocket launcher is expounded, which, based on fuzzy clustering, helps realizing application of uncertain or insufficient knowledge of human in subjective and practical experiences, so as to succeed in the most accurate classification determination for types of fault thereon. During rocket launcher drilling of the army, type of fault can be determined accurately and quickly with this method in case of fault, and the operator can remove such fault quickly by adopting appropriate method in terms of type of fault, which greatly improves technical supporting capacity of rocket launcher and generates remarkable economic and military benefits.

\section{References}

[1] Brandt M,Kharas Y.An error convergence simulation study of hardvs.fuzzy C-means clustering. In:IEEEInter-NationalConference on Fuzzy System.Orlando:The IEEE Neural Networks Council and the IEEE Orlando Section,1994.1835-1839

[2] Sam J,Chan Laiwan.Alternative membership function for sequential fuzzy clustering.In:IEEE International Con-Ferenc on Fuzzy System.Orlando:The IEEE Neural Networks Council and the IEEE Orlando Section,1994.1846-1851

[3] Wang Shuhai et al, Mechanism of Rocket Launcher [M]; Beijing: Chinese People's Liberation Army Publishing House, 2000

[4] Xu Zhangsui, Fang Liqing, Wang Xiwu et al, Fundamental \& Application of Fault Information Diagnosis, [M]; Beijing: National defense Industry Press, 2000

[5] Li Hongxing, Wang Qun, Duan Qinzhi et al, Methodology \& Application of Engineering Fuzzy Mathematics [M]; Tianjin: Tianjin Science and Technology Press, 1993

[6] Wu Jinpei, Fuzzy Diagnosis Theory and Application [M]; Beijing: Science Press, 1995

[7] Gao Xinbo, Fuzzy Clustering Analysis and Application [M]; Xi'an: Xi'an University of Electronic Science and Technology Press, 2004 